\title{
Risk factor analysis of proximal junctional kyphosis after posterior osteotomy in patients with ankylosing spondylitis
}

\author{
*Tianhao Wang, PhD, ${ }^{1,2}$ Yongfei Zhao, PhD, ${ }^{2}$ Yan Liang, $\mathrm{PhD},{ }^{2}$ Haocong Zhang, PhD, ${ }^{2}$ \\ Zheng Wang, $\mathrm{PhD},{ }^{2}$ and Yan Wang, $\mathrm{PhD}^{2}$ \\ ${ }^{1}$ Southwest Hospital, Third Military Medical University, Chongqing; and 2Department of Orthopaedics, General Hospital of the \\ Chinese People's Liberation Army, Beijing, China
}

\begin{abstract}
OBJECTIVE The aim of this paper was to analyze the incidence and risk factors of proximal junctional kyphosis (PJK) in patients with ankylosing spondylitis (AS) who underwent pedicle subtraction osteotomy.

METHODS The records of 83 patients with AS and thoracolumbar kyphosis who underwent surgery at the authors' institution between 2007 and 2013 were reviewed. The patients were divided into 2 groups based on the presence or absence of PJK. The radiographic measurements, including proximal junctional angle (PJA), sagittal parameters, and pelvic parameters of these 2 groups, were compared at different time points: before surgery and 2 weeks, 12 months, and 2 years after surgery. Oswestry Disability Index scores were also evaluated.
\end{abstract}

RESULTS Overall, $14.5 \%$ of patients developed PJK. Before surgery, the mean PJAs in the 2 groups were $13.6^{\circ}$ and $8.5^{\circ}$, respectively $(p=0.008)$. There were no significant differences in age, sex, and body mass index between groups. Patients with PJK had a larger thoracolumbar kyphotic angle $\left(50.8^{\circ} \pm 12.6^{\circ}\right)$ and a greater sagittal vertical axis $(21.7 \pm$ $4.3 \mathrm{~cm}$ ) preoperatively than those without PJK. The proportion of patients with PJK whose fusion extended to the sacrum was $41.2 \%(7 / 17)$, which is significantly greater than the proportion of patients with PJK whose lowest instrumented vertebra was above the sacrum. Oswestry Disability Index scores did not significantly increase in the PJK group compared with the non-PJK group.

CONCLUSIONS The authors found that PJK occurs postoperatively in patients with AS with an incidence of $14.5 \%$. Risk factors of PJK include larger preoperative sagittal vertical axis, PJA, and osteotomy angle. Reducing the osteotomy angle in some severe cases and extending fusion to a higher, flatter level would be also beneficial in decreasing the risk of PJK.

https://thejns.org/doi/abs/10.3171/2017.11.SPINE17228

KEYWORDS ankylosing spondylitis; proximal junctional kyphosis; complication; risk factors; deformity

$\mathrm{A}$ NKYLOSING spondylitis (AS) is a chronic inflammatory disease that eventually results in a rigid thoracolumbar kyphotic deformity, which can lead to an impairment of horizontal gaze in the late stage. ${ }^{2}$ The surgical treatment for the disease is spinal osteotomy. In the past, most studies of complications associated with AS have concentrated on gastrointestinal and neurological complications. ${ }^{1,16} \mathrm{Few}$ investigators have focused on internal fixation complications.

Proximal junctional kyphosis (PJK) is an important complication after long segment fusion. There are 2 gen- erally accepted definitions of PJK: 1) a kyphotic angle of greater than $20^{\circ}$ between the upper instrumented vertebra (UIV) and 2 levels above the UIV,${ }^{15}$ and 2 ) a proximal junctional angle $(\mathrm{PJA}) \geq 10^{\circ}$ greater than the preoperative measurements. ${ }^{4}$ The incidence of PJK has been reported to range from $9 \%$ to $46 \%$ in adolescent idiopathic scoliosis (AIS) and from $26 \%$ to $41 \%$ in adult spinal deformity (ASD) ${ }^{4,8,18,19}$ In recent years, a growing number of surgeons have begun to pay attention to PJK because of the severe postoperative clinical outcomes.

To date, a large number of studies have focused on PJK

ABBREVIATIONS AIS = adolescent idiopathic scoliosis; $A S=$ ankylosing spondylitis; $A S D=$ adult spinal deformity; BMI = body mass index; LIV = lowest instrumented vertebra; $\mathrm{LL}=$ lumbar lordosis; $\mathrm{OA}=$ osteotomy angle; $\mathrm{ODI}=$ Oswestry Disability Index; $\mathrm{PI}=$ pelvic incidence; $\mathrm{PJA}=$ proximal junctional angle; $\mathrm{PJK}=$ proximal junctional kyphosis; PSO = pedicle subtraction osteotomy; SVA = sagittal vertical axis; TK = thoracic kyphosis; TLK = thoracolumbar kyphosis; UIV = upper instrumented vertebra. SUBMITTED March 19, 2017. ACCEPTED November 16, 2017.

INCLUDE WHEN CITING Published online April 20, 2018; DOI: 10.3171/2017.11.SPINE17228.

* T.W. and Y.Z. contributed equally to this work and share first authorship. 
occurring in mobile junction areas. In a recent case report, the authors suggested that PJK might also occur in the ankylosing spine. ${ }^{6}$ In AS patients, similarly, the spine is rigid and unmovable. Whether patients with AS will experience PJK remains unclear. Thus, the purpose of this study was to observe the incidence of PJK in AS patients who underwent pedicle subtraction osteotomy (PSO) and analyze the probable risk factors that contribute to the development of PJK.

\section{Methods}

We performed a retrospective analysis of 134 patients with AS who underwent spine surgery at the Chinese PLA General Hospital between 2007 and 2013. The records were reviewed to meet the following inclusion criteria: patients who were diagnosed with AS based on modified New York criteria $^{17}$ and underwent treatment of their thoracolumbar kyphosis (TLK) via a 1-level or interrupted 2-level PSO, thoracic or thoracolumbar (T-5 to L-2) UIV, minimum 2-year follow-up, and clear identification of the proximal junctional vertebra on lateral radiographs. Patients who underwent revision surgery because of infection, cerebrospinal fluid leak, nerve injury, or rod breakage at the osteotomy site were excluded from the study. Therefore, this study included 83 patients (76 males and 7 females) with a mean age of 35.2 years (range $23-57$ years). The mean follow-up was 28.4 months (range 24-54 months).

The patients were classified into 2 groups based on the presence or absence of PJK. PJK in this study was defined as a $\mathrm{PJA} \geq 20^{\circ}$ or an angle $\geq 10^{\circ}$ greater than the preoperative measurement. ${ }^{4}$

The imaging data were obtained before and 2 weeks after surgery; at 6 months, 12 months, and 2 years postoperatively; and at final follow-up. The indication for revision surgery was according to the Hart-ISSG PJK Severity Scale. ${ }^{11}$ The radiographic parameters included PJA (the angle between the UIV and 2 levels above the UIV), thoracic kyphosis (TK; the angle between the superior endplate of T-5 and inferior endplate of T-12), TLK (the angle between the superior endplate of T-10 and inferior endplate of L-2), and lumbar lordosis (LL, the angle between the superior endplate of L-1 and S-1). Sagittal vertical axis (SVA) was measured as the distance between the C-7 plumb line and the superior posterior corner of S-1. Pelvic incidence (PI) was measured from the angle subtended by a perpendicular from the cephalic endplate of S-1 and a line connecting the center of the femoral head to the center of the cephalic endplate of S-1. The osteotomy angle (OA), that is, the angle between the upper endplate of 1 vertebra above the osteotomy site and the lower endplate 1 vertebra below, was also recorded. The OA in interrupted 2-level osteotomy cases was the sum of the angles of each osteotomy segment. All measurements were performed by a radiologist and a spine surgeon with the aid of Surgimap version 2.2, and the average values were recorded. Clinical data included age, sex, and body mass index (BMI). The Oswestry Disability Index (ODI) was used to evaluate patient outcomes.

\section{Statistical Analysis}

The analyses were performed using IBM SSPS (version
22, IBM Corp.); $\mathrm{p}<0.05$ was considered significant. Continuous variables were presented as means and standard deviations and compared with the Student t-test, while categorical variables were compared with the chi-square test.

\section{Results}

Among the included 83 patients, 71 patients (83.6\%) had normal proximal kyphosis during the follow-up period, and 12 (14.5\%) were deemed as having PJK (Fig. 1). In the PJK group, 9 patients experienced PJK within 6 months after surgery and 3 patients developed PJK between 6 and 12 months after surgery. No patients developed PJK after 12 months postsurgery.

The baseline patient characteristics are shown in Table 1. We found that age, sex, and BMI were similar between the groups (all $\mathrm{p}>0.05$ ). In addition, the number of fusion segments did not significantly differ between the groups (Table 1). Before surgery, the mean ODI scores of the PJK and non-PJK groups were $32.9 \pm 8.2$ and $34.7 \pm 7.9$. At the final follow-up, the scores significantly decreased to $7.2 \pm$ 3.3 and $7.5 \pm 3.7$, respectively $(p=0.012)$. However, there was no significant difference between the groups preoperatively or postoperatively $(\mathrm{p}>0.05)$. Surgical characteristics are listed in Table 2.

Table 3 lists the radiographic characteristics of the patients. The PJA in these 2 groups increased during followup. Compared with the patients in the non-PJK group, more patients in the PJK group had a larger PJA ( $\mathrm{p}=$ $0.008)$, greater TK $(p=0.002)$, greater $\mathrm{OA}(\mathrm{p}<0.001)$, and greater preoperative SVA $(p=0.009)$. The OA was greater in the PJK group than in the non-PJK group $\left(61.8^{\circ} \pm 6.3^{\circ}\right.$ vs $\left.45.1^{\circ} \pm 10.5^{\circ}, \mathrm{p}<0.001\right)$. However, the differences between the preoperative and postoperative values for pelvic tilt, LL, PI, and sagittal slope were not statistically significant between the 2 groups (Table 3 ).

The proportion of patients with the UIV at T-9 or above who experienced PJK postoperatively was $36 \%(9 / 25)$, which was significantly higher than that in patients with the UIV below T-9 $(5 \%, 3 / 58 ; \mathrm{p}<0.001)$. The choice of lowest instrumented vertebra (LIV) at S-1 led to $41.2 \%(7 / 17)$ patients developing PJK, which was higher than LIV above L-5 $(10.0 \%, 1 / 10)$ and LIV at L-5 $(7.1 \%, 4 / 56 ; p=0.002)$. Additionally, there was no difference between single-level osteotomy and interrupted 2-level osteotomy (Table 2).

\section{Discussion}

Previous studies have suggested that PJK might be caused by destruction of the posterior ligamentous complex and stress changes in the proximal junctional region after long segment spinal fusion. ${ }^{19}$ In spinal osteotomy for AS patients, long fixation of the spine is helpful in terms of postoperative spinal stability. In recent years, several studies have reported PJK occurring in patients with AS, and PJK was identified in an ankylosing area in a report by Ikegami et al. ${ }^{6}$ Systematic studies analyzing the incidence and risk factors of PJK in AS patients are lacking.

\section{Incidence of PJK}

Zhu et al. ${ }^{21}$ observed 50 AS patients for a minimum of 

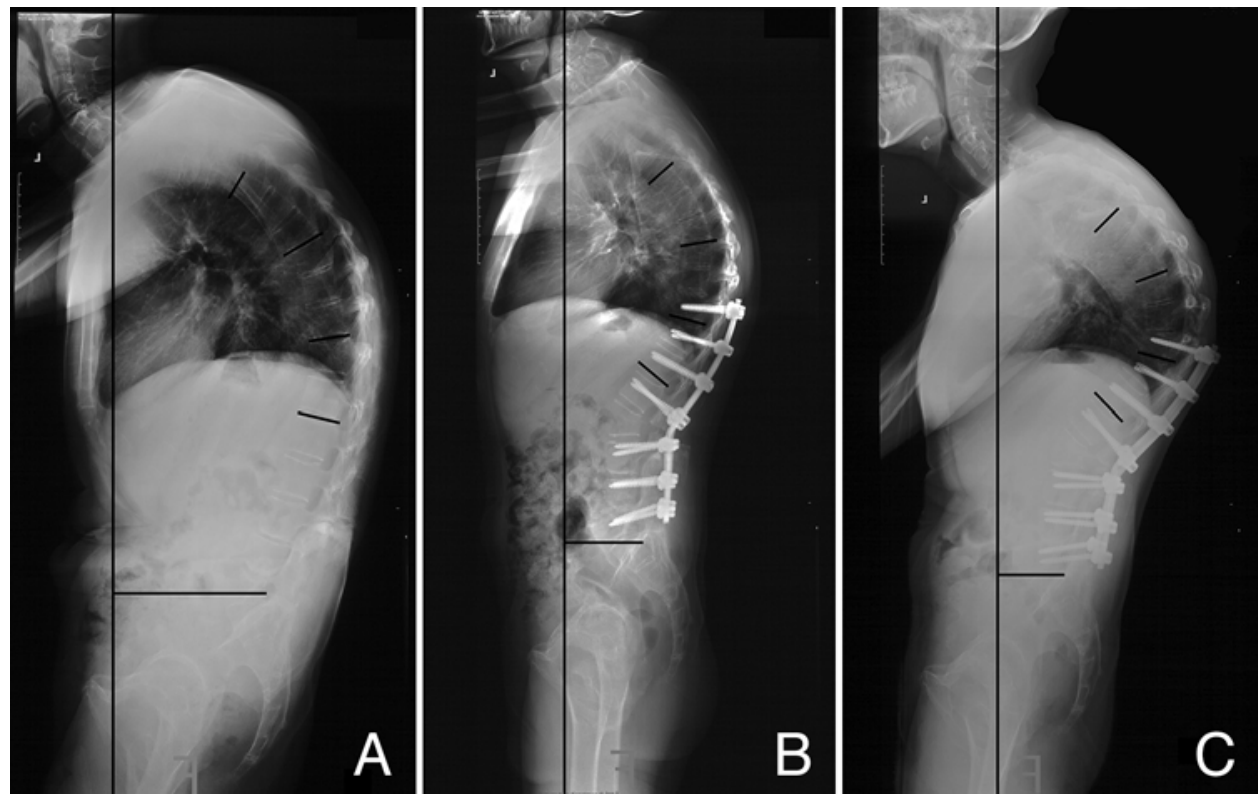

FIG. 1. Lateral radiographs obtained in a 34-year-old man who had undergone posterior osteotomy and instrumented fusion (T10L5) for the treatment of AS and subsequently developed PJK. A: Preoperatively, the PJA, TK, and SVA were $22^{\circ}, 79^{\circ}$, and 14.0 $\mathrm{cm}$, respectively. B: Three months postoperatively, the PJA, TK, and SVA were $24^{\circ}, 72^{\circ}$, and $8.4 \mathrm{~cm}$, respectively. C: Two years postoperatively, the PJA, TK, and SVA were $35^{\circ}, 83^{\circ}$, and $6.7 \mathrm{~cm}$, respectively.

2 years who had undergone Smith-Petersen osteotomy or PSO, and PJK occurred in 3 cases $(6.0 \%)$. In the current study, 12 of the 83 patients (14.5\%) experienced PJK during follow-up, and the PJA was found to increase at each follow-up. Wang et al. ${ }^{18}$ reported that $80 \%$ of patients who underwent surgical treatment for idiopathic scoliosis experienced PJK within 1.5 years of surgery. Similarly, in the current study, $12.3 \%$ of patients experienced PJK within 6 months postoperatively, and $4.1 \%$ of patients experienced PJK at 1 year after surgery, with no further increases thereafter. Nevertheless, in the non-PJK group, the PJA was also slightly increased. Although these cases did not satisfy the criteria of PJK, long-term follow-up is still recommended.

\section{Analysis of PJK Factors}

Authors have reported various opinions on the risk factors for the development of PJK because of different research objectives, types of operation, and follow-up times. According to Kim and Iyer, ${ }^{7}$ the etiology of PJK can be di-

TABLE 1. Demographic and baseline information of patients with and without PJK after undergoing surgery for AS

\begin{tabular}{lrcc}
\hline \multicolumn{1}{c}{ Variable } & Non-PJK & PJK & p Value \\
\hline Mean age (yrs) & $35.1 \pm 9.7$ & $35.5 \pm 7.5$ & 0.898 \\
\hline Sex (no. of patients) & & & 0.565 \\
\hline F & 7 & 0 & \\
\hline M & 64 & 12 & \\
\hline Mean BMI $\left(\mathrm{kg} / \mathrm{m}^{2}\right)$ & $27.7 \pm 5.5$ & $28.2 \pm 9.2$ & 0.835 \\
\hline Mean no. of fusion segments & $7.7 \pm 1.3$ & $9.4 \pm 0.8$ & 0.081 \\
\hline
\end{tabular}

Mean values are presented \pm SD. vided into surgical, radiographic, and patient-related risk factors. Surgical factors mainly include destruction of the posterior ligamentous complex, choice of UIV, and fusion to the sacrum. Radiographic factors include large SVA, TK, LL, PI, or PJA, and smaller sagittal slope preoperatively. Patient-related factors, such as age and BMI, also contribute to the development of PJK.

\section{Surgical Risk Factors}

Fusion to lower lumbar levels or the sacrum is closely associated with PJK in AIS and ASD patients. ${ }^{18,19}$ Researchers have found that patients' coronal and sagittal plane balance may benefit from preserving the motion of

TABLE 2. Surgical characteristics for patients with and without PJK

\begin{tabular}{cccc}
\hline Variable & Non-PJK & PJK & p Value \\
\hline Osteotomy selection & & & 0.465 \\
\hline 1 level & 49 & 7 & \\
\hline Interrupted 2 levels & 22 & 5 & \\
\hline Distribution of UIV & & & $<0.001^{*}$ \\
\hline T-9 \& above & 16 & 9 & \\
\hline Below T-9 & 55 & 3 & \\
\hline Distribution of LIV & & & $0.002^{*}$ \\
\hline Above L-5 & 9 & 1 & \\
\hline L-5 & 52 & 4 & \\
\hline S-1 & 10 & 7 & \\
\hline
\end{tabular}

Values are number of patients unless otherwise indicated.

* Statistically significant. 
TABLE 3. Time-dependent change of the radiographic parameters and outcomes

\begin{tabular}{|c|c|c|c|}
\hline Variable & Non-PJK Group & PJK Group & $p$ Value \\
\hline \multicolumn{4}{|l|}{$\operatorname{PJA}\left({ }^{\circ}\right)$} \\
\hline Preop & $8.5 \pm 3.5$ & $13.6 \pm 1.6$ & $0.008^{*}$ \\
\hline Immediately postop & $9.2 \pm 3.0$ & $15.7 \pm 3.2$ & $<0.001^{*}$ \\
\hline Final follow-up & $10.4 \pm 5.1$ & $23.7 \pm 4.3$ & $<0.001^{*}$ \\
\hline \multicolumn{4}{|l|}{$\mathrm{TK}\left({ }^{\circ}\right)$} \\
\hline Preop & $42.1 \pm 13.6$ & $79.5 \pm 12.9$ & $0.002^{*}$ \\
\hline Immediately postop & $42.9 \pm 9.4$ & $48.8 \pm 8.7$ & 0.110 \\
\hline Final follow-up & $41.8 \pm 11.2$ & $53.4 \pm 13.1$ & 0.245 \\
\hline \multicolumn{4}{|l|}{$\operatorname{TLK}\left({ }^{\circ}\right)$} \\
\hline Preop & $46.5 \pm 14.4$ & $50.8 \pm 12.6$ & 0.063 \\
\hline Immediately postop & $7.4 \pm 14.5$ & $5.1 \pm 14.7$ & 0.400 \\
\hline Final follow-up & $5.8 \pm 19.7$ & $7.4 \pm 9.1$ & 0.672 \\
\hline \multicolumn{4}{|l|}{$\operatorname{LL}\left({ }^{\circ}\right)$} \\
\hline Preop & $-28.9 \pm 23.3$ & $-23.6 \pm 28.0$ & 0.547 \\
\hline Immediately postop & $-40.3 \pm 12.4$ & $-48.3 \pm 15.8$ & 0.092 \\
\hline Final follow-up & $-42.1 \pm 14.2$ & $-53.5 \pm 13.3$ & 0.115 \\
\hline \multicolumn{4}{|l|}{$\mathrm{PT}\left({ }^{\circ}\right)$} \\
\hline Preop & $38.3 \pm 8.3$ & $38.2 \pm 15.4$ & 0.075 \\
\hline Immediately postop & $23.5 \pm 8.2$ & $21.8 \pm 10.7$ & 0.736 \\
\hline Final follow-up & $26.6 \pm 9.8$ & $22.9 \pm 8.5$ & 0.063 \\
\hline \multicolumn{4}{|l|}{$\operatorname{SS}\left({ }^{\circ}\right)$} \\
\hline Preop & $8.9 \pm 12.0$ & $12.6 \pm 6.5$ & 0.313 \\
\hline Immediately postop & $22.9 \pm 6.9$ & $29.2 \pm 10.6$ & 0.053 \\
\hline Final follow-up & $21.0 \pm 7.8$ & $24.9 \pm 5.1$ & 0.092 \\
\hline \multicolumn{4}{|l|}{ SVA (cm) } \\
\hline Preop & $12.0 \pm 8.7$ & $21.7 \pm 4.3$ & $0.009^{*}$ \\
\hline Immediately postop & $8.6 \pm 5.1$ & $12.1 \pm 6.0$ & 0.614 \\
\hline Final follow-up & $8.8 \pm 4.1$ & $11.1 \pm 2.2$ & 0.833 \\
\hline \multicolumn{4}{|l|}{ ODI } \\
\hline Preop & $32.9 \pm 8.2$ & $34.7 \pm 7.9$ & 0.328 \\
\hline Final follow-up & $7.2 \pm 3.3$ & $7.5 \pm 3.7$ & 0.281 \\
\hline $\mathrm{PI}\left({ }^{\circ}\right)$ & $47.2 \pm 7.1$ & $50.6 \pm 11.2$ & 0.612 \\
\hline $\mathrm{OA}\left({ }^{\circ}\right)$ & $45.1 \pm 10.5$ & $61.8 \pm 6.3$ & $<0.001^{*}$ \\
\hline
\end{tabular}

PT = pelvic tilt; SS = sacral slope.

Mean values are presented \pm SD .

* Statistically significant.

distal segments. In AS patients, the data revealed that patients with fusion to the sacrum had higher risk of PJK. However, fusion to the sacrum may not be a major factor for the development of PJK. In this study, most patients who had fusion to the sacrum had severe spine kyphosis and sagittal imbalance (larger preoperative SVA) and required an interrupted 2-level osteotomy at the L-3 or L-4 level. Thus, the selection of LIV is closely related to the surgical plan and is more a reflection of preoperative sagittal plane deformity than a risk factor of PJK. However, we suggest that S-1 is not the optimal LIV in most cases. For one, patients with S-1 fixation tend to suffer more severe lumbosacral pain. ${ }^{20}$ For another, implantation of screws at
S-1 increases the operative time but does not increase the stability of internal fixation because the fusion between L-5 and S-1 is stable.

Lau et al. verified that greater curvature correction is one of the major risk factors for PJK, which was in line with our results. ${ }^{11}$ Reconstructing sagittal balance is the main purpose of surgery for the kyphotic deformity. However, field of vision also affects quality of life and must also be taken into consideration. As a result, there should be a tradeoff between SVA correction and horizontal gaze, and thus the OA should be decreased to guarantee a more horizontal visual field. In most cases, patients with interrupted 2-level osteotomy undergo greater correction than those undergoing 1-level osteotomy. Although the occurrence of PJK in the former group (2-level group, 18.5\%) was higher than that in the latter group (1-level group, $12.5 \%$ ), this difference was not statistically significant. A paired study with a larger sample size might produce more meaningful results.

The choice of UIV also affects the occurrence of PJK. In AS patients, a UIV in the thoracolumbar area appears to be associated with a reduced risk of PJK, although it might cause vertebral fracture in patients with ASD. ${ }^{14}$ In most AS cases, the thoracolumbar and lumbar regions are most affected by kyphosis, and L-2 or L-3 is recommended as the osteotomy site. ${ }^{20}$ A UIV above T-9 is a result of a higher osteotomy site. Patients with a UIV above the thoracolumbar region tend to have more severe TK and a larger preoperative SVA, which might indicate that UIV is not independently associated with PJK.

\section{Radiographic Risk Factors}

In AIS and ASD, preoperative PJK is often associated with the occurrence of PJK postoperatively. We draw a similar conclusion in this study that a larger preoperative sagittal angle in the proximal junctional region increases the risk of PJK developing postoperatively. In ASD, a larger PJA is associated with PJK and might lead to fracture at the UIV.12 In the rigid spine of AS patients, a larger sagittal angle of proximal junctional segments leads to stress concentration, which may lead to PJK aggravation or even proximal junctional failure. Despite the fact that we did not observe fracture or fixation failure at the UIV in AS patients, we still suggest that extending the fusion to a higher level in patients with preexisting PJK will decrease the risk of postoperative PJK.

As for sagittal parameters, we found that large preoperative TK and SVA are closely associated with PJK. Sagittal balance is the most important factor that should be taken into consideration in operative planning. Surgery can lessen the sagittal imbalance by correcting local kyphosis, but curvature in the thoracic area will remain postoperatively. Furthermore, in most cases surgery cannot correct the SVA to within the normal range $(<5 \mathrm{~cm})$. In some severe cases, the SVA cannot be corrected to within a normal range because of the potential risk of the patient losing the ability to look straight ahead. Indeed, cervical osteotomy is one solution that can provide a horizontal visual field, but it is not recommended because of the difficulty of the operation and the high risk of neurological complications. Rather than taking these risks, our prefer- 
ence is to perform less correction of sagittal balance to ensure that a patient's visual field is not affecting daily life. Thus, postoperative sagittal imbalance remains, which might be a reason that PJK develops.

\section{Patient-Related Risk Factors}

We found that patient age is not a significant factor in the occurrence of PJK. Past research has indicated that patients older than 55 years are at higher risk for PJK. ${ }^{10}$ Degenerative changes of intervertebral discs, facet joints, and paravertebral muscles are the factors associated with aging that most commonly contribute to the development of PJK. In AS, however, those structures are rigid and immobile, which might decrease the effect of tissue degeneration on patients in different age groups.

BMI was reported by Bridwell et al. ${ }^{3}$ and O'Leary et al. ${ }^{13}$ as a risk factor for PJK. However, we were unable to find the same association in our study. BMI is regarded as a reliable tool for predicting total body fat. However, considering that AS patients have severe kyphosis, BMI cannot accurately reflect the degree of obesity. In AS patients, the ankylosed spine can act on the long lever arm and result in a concentration of junctional stress. ${ }^{6}$ The increased upper body weight in heavy patients might influence the lever effect and result in PJK. Preoperative BMI cannot serve as a predictive index for PJK. A new index that takes into account postoperative BMI, SVA, and kyphotic angle of the upper spine of the internal fixation may be more reliable. Thus, future research should better adopt a more suitable index to assess the relationship between PJK and obesity in AS patients.

\section{Clinical Outcomes}

Our research indicates that AS patients with PJK do not experience more pain than those without PJK; no patient experienced proximal junctional failure during the followup, results that are similar to those of AIS. ${ }^{9}$ However, it has been reported that in some ASD patients PJK can be more severe, and revision surgery might be required. ${ }^{5}$ Although PJK has little effect on patient outcome, it still requires special attention. For one, the PJA continued to increase after surgery. Proximal junctional failure occurring in the ankylosing spine might cause severe fracture instability and cord deficit. ${ }^{6}$ For another, AS patients are often younger (mean age of 35.2 years in this study) when undergoing surgery, and the disease may still be active, resulting in aggravation of spinal kyphosis.

There are a number of limitations to our study. Some other factors associated with PJK, such as bone mineral density, vertebroplasty, and spinal fixation modes, were not included in the study. Bone mineral density was not routinely assessed, and therefore that information is lacking. A posterior pedicle screw system was used in every patient, resulting in a lack of cases for comparison. In further studies, these factors will be examined, and longer follow-up time will be adopted. We also did not assess the degree of spinal stiffness. To measure this factor, sagittal and coronal bending radiographs of the spine are needed. This information would be helpful in understanding the unique aspect of PJK in the AS patient population.

\section{Conclusions}

We found that PJK occurs in AS patients after long segment fusion with an incidence of $14.5 \%$. The 3 aspects that may affect the incidence of PJK are 1) greater preoperative sagittal imbalance, 2) greater local kyphosis at proximal junctional area, and 3) greater curvature correction. In other words, preoperative SVA, PJA, and OA are closely associated with the occurrence of PJK. Reducing the OA in some severe cases will not only ensure patients a more normal visual field but also decrease the risk of PJK. Extending fusion to a higher, flatter level might also be beneficial in avoiding this problem.

\section{References}

1. Arun R, Dabke HV, Mehdian H: Comparison of three types of lumbar osteotomy for ankylosing spondylitis: a case series and evolution of a safe technique for instrumented reduction. Eur Spine J 20:2252-2260, 2011

2. Braun J, Sieper J: Ankylosing spondylitis. Lancet 369:1379_ 1390, 2007

3. Bridwell KH, Lenke LG, Cho SK, Pahys JM, Zebala LP, Dorward IG, et al: Proximal junctional kyphosis in primary adult deformity surgery: evaluation of 20 degrees as a critical angle. Neurosurgery 72:899-906, 2013

4. Glattes RC, Bridwell KH, Lenke LG, Kim YJ, Rinella A, Edwards C II: Proximal junctional kyphosis in adult spinal deformity following long instrumented posterior spinal fusion: incidence, outcomes, and risk factor analysis. Spine (Phila Pa 1976) 30:1643-1649, 2005

5. Hart RA, McCarthy I, Ames CP, Shaffrey CI, Hamilton DK, Hostin R: Proximal junctional kyphosis and proximal junctional failure. Neurosurg Clin N Am 24:213-218, 2013

6. Ikegami D, Matsuoka T, Miyoshi Y, Murata Y, Aoki Y: Proximal junctional failure after long-segment instrumentation for degenerative lumbar kyphosis with ankylosing spinal disorder. Spine (Phila Pa 1976) 40:E740-E743, 2015

7. Kim HJ, Iyer S: Proximal junctional kyphosis. J Am Acad Orthop Surg 24:318-326, 2016

8. Kim HJ, Lenke LG, Shaffrey CI, Van Alstyne EM, Skelly AC: Proximal junctional kyphosis as a distinct form of adjacent segment pathology after spinal deformity surgery: a systematic review. Spine (Phila Pa 1976) 37 (22 Suppl):S144S164, 2012

9. Kim HJ, Yagi M, Nyugen J, Cunningham ME, Boachie-Adjei $\mathrm{O}$ : Combined anterior-posterior surgery is the most important risk factor for developing proximal junctional kyphosis in idiopathic scoliosis. Clin Orthop Relat Res 470:16331639,2012

10. Kim YJ, Lenke LG, Bridwell KH, Kim J, Cho SK, Cheh G, et al: Proximal junctional kyphosis in adolescent idiopathic scoliosis after 3 different types of posterior segmental spinal instrumentation and fusions: incidence and risk factor analysis of 410 cases. Spine (Phila Pa 1976) 32:2731-2738, 2007

11. Lau D, Clark AJ, Scheer JK, Daubs MD, Coe JD, Paonessa KJ, et al: Proximal junctional kyphosis and failure after spinal deformity surgery: a systematic review of the literature as a background to classification development. Spine (Phila Pa 1976) 39:2093-2102, 2014

12. Maruo K, Ha Y, Inoue S, Samuel S, Okada E, Hu SS, et al: Predictive factors for proximal junctional kyphosis in long fusions to the sacrum in adult spinal deformity. Spine (Phila Pa 1976) 38:E1469-E1476, 2013

13. O'Leary PT, Bridwell KH, Lenke LG, Good CR, Pichelmann MA, Buchowski JM, et al: Risk factors and outcomes for catastrophic failures at the top of long pedicle screw constructs: 
a matched cohort analysis performed at a single center. Spine (Phila Pa 1976) 34:2134-2139, 2009

14. O'Shaughnessy BA, Bridwell KH, Lenke LG, Cho W, Baldus C, Chang MS, et al: Does a long-fusion "T3-sacrum" portend a worse outcome than a short-fusion "T10-sacrum" in primary surgery for adult scoliosis? Spine (Phila Pa 1976) 37:884-890, 2012

15. Scheer JK, Fakurnejad S, Lau D, Daubs MD, Coe JD, Paonessa KJ, et al: Results of the 2014 SRS Survey on PJK/PJF: a report on variation of select SRS member practice patterns, treatment indications, and opinions on classification development. Spine (Phila Pa 1976) 40:829-840, 2015

16. Sugrue PA, O'Shaughnessy BA, Nasr F, Koski TR, Ondra SL: Abdominal complications following kyphosis correction in ankylosing spondylitis. J Neurosurg Spine 10:154-159, 2009

17. van der Linden S, Valkenburg HA, Cats A: Evaluation of diagnostic criteria for ankylosing spondylitis. A proposal for modification of the New York criteria. Arthritis Rheum 27:361-368, 1984

18. Wang J, Zhao Y, Shen B, Wang C, Li M: Risk factor analysis of proximal junctional kyphosis after posterior fusion in patients with idiopathic scoliosis. Injury 41:415-420, 2010

19. Yagi M, Akilah KB, Boachie-Adjei O: Incidence, risk factors and classification of proximal junctional kyphosis: surgical outcomes review of adult idiopathic scoliosis. Spine (Phila Pa 1976) 36:E60-E68, 2011

20. Yao Z, Zheng G, Zhang Y, Wang Z, Zhang X, Cui G, et al:
Selection of lowest instrumented vertebra for thoracolumbar kyphosis in ankylosing spondylitis. Spine (Phila Pa 1976) 41:591-597, 2016

21. Zhu Z, Wang X, Qian B, Wang B, Yu Y, Zhao Q, et al: Loss of correction in the treatment of thoracolumbar kyphosis secondary to ankylosing spondylitis: a comparison between Smith-Petersen osteotomies and pedicle subtraction osteotomy. J Spinal Disord Tech 25:383-390, 2012

\section{Disclosures}

The authors report no conflict of interest concerning the materials or methods used in this study or the findings specified in this paper.

\section{Author Contributions}

Conception and design: Y Wang. Acquisition of data: Liang, Zhang. Analysis and interpretation of data: Zhao, Liang. Drafting the article: $\mathrm{T}$ Wang. Critically revising the article: Y Wang, Z Wang. Reviewed submitted version of manuscript: all authors. Administrative/technical/material support: Y Wang. Study supervision: Y Wang, Z Wang.

\section{Correspondence}

Yan Wang: The General Hospital of the Chinese People's Liberation Army, Beijing, China. yanwang_301@126.com. 